\title{
Dynamics stabilization and transport coherency in a rocking ratchet for cold atoms
}

\author{
A.B. Kolton ${ }^{1}$ and F. Renzoni ${ }^{2}$ \\ ${ }^{1}$ CONICET, Centro Atomico Bariloche, 8400 San Carlos de Bariloche, Rio Negro, Argentina and \\ ${ }^{2}$ Department of Physics and Astronomy, University College London, \\ Gower Street, London WC1E 6BT, United Kingdom
}

(Dated: June 23, 2018)

\begin{abstract}
Cold atoms in optical lattices have emerged as an ideal system to investigate the ratchet effect, as demonstrated by several recent experiments. In this work we analyze theoretically two aspects of ac driven transport in cold atoms ratchets. We first address the issue of whether, and to which extent, an ac driven ratchet for cold atoms can operate as a motor. We thus study theoretically a dissipative motor for cold atoms, as obtained by adding a load to a $1 \mathrm{D}$ non-adiabatically driven rocking ratchet. We demonstrate that a current can be generated also in the presence of a load, e.g. the ratchet device can operate as a motor. Correspondingly, we determine the stall force for the motor, which characterizes the range of loads over which the device can operate as a motor, and the differential mobility, which characterizes the response to a change in the magnitude of the load. Second, we compare our results for the transport in an ac driven ratchet device with the transport in a dc driven system. We observe a peculiar phenomenon: the bi-harmonic ac force stabilizes the dynamics, allowing the generation of uniform directed motion over a range of momentum much larger than what is possible with a dc bias. We explain such a stabilization of the dynamics by observing that a non-adiabatic ac drive broadens the effective cooling momentum range, and forces the atom trajectories to cover such a region. Thus the system can dissipate energy and maintain a steady-state energy balance. Our results show that in the case of a finite-range velocity-dependent friction, a ratchet device may offer the possibility of controlling the particle motion over a broader range of momentum with respect to a purely biased system, although this is at the cost of a reduced coherency.
\end{abstract}

PACS numbers:

\section{INTRODUCTION}

The ratchet effect [1-7] is usually defined as the rectification of fluctuations in the absence of a bias, as obtained in a system out of equilibrium. This typically corresponds to the generation of directed transport of particles through a macroscopically flat periodic structure.

The concept of ratchet leads naturally to the idea of Brownian motor, i.e. to the possibility of doing work against a load as a result of the rectification of fluctuations. Any ratchet device can then be characterized as a motor [8 11], thus pointing out the features related to the possibility of doing work against a load.

Cold atoms in optical lattices have emerged as an ideal system to investigate the ratchet effect [12, 13]. The significant advantage of this system is tunability, which allows for arbitrary periodic potentials to be created, and drivings to be applied either under the form of rocking forces or as modulation of the potential amplitude. This allowed for the realization of $1 \mathrm{D}$ rocking ratchets, both with periodic [14 16] and quasiperiodic driving [17], and gating ratchets [18]. Furthermore, rectification mechanisms unique to higher-dimensional systems were demonstrated by using 2D rocking ratchets [19]. Despite the number of successful realizations of driven ratchets for cold atoms, some fundamental aspects of the transport in these devices have yet to be analyzed. A first important issue is whether cold atom ratchets can, and to which extent, operate as motors, e.g. can do positive work against a load. A second fundamental issue is how the transport in cold atom ratchets compare with the transport as obtained by the direct application of a dc bias. This work precisely adresses these issues. First, we study and characterize theoretically the motor corresponding to a $1 \mathrm{D}$ rocking ratchet for cold atoms, as obtained by adding a load to the ratchet set-up. We demonstrate that a current can be generated also in the presence of a load, e.g. the ratchet device can operate as a motor. Correspondingly, we determine the stall force for the motor, which characterizes the range of loads over which the device can operate as a motor, and the differential mobility, which characterizes the response to a change in the magnitude of the load. Second, we compare transport in an ac driven ratchet device with the transport in a dc driven system. A quantitative study is performed, by determining the magnitude of the obtained velocity, as well as the coherency of the directed transport. It turns out that the ratchet set-up allows to generate uniform motion over a range of velocities larger than in the case of the dc driven system. An explanation for this stabilization phenomenon for ac driven transport is given.

This work is organized as follows. In Sec. II we describe the ratchet set-up. In Sec. III we first characterize a motor for cold atoms, as obtained by adding a load to an ac driven ratchet. We thus present our numerical results for the stall force and the differential mobility. We then determine the coherence of transport in an unbiased ratchet, and compare our results to a system of cold 
atoms in an optical lattice with a pure dc driving force. The observed stabilization phenomenon is discussed and analyzed. In Sec. IV conclusions are drawn.

\section{RATCHET SET-UP}

The ratchet set-up considered in this work is the same as the one examined in Ref. [20]. A dissipative optical lattice is created by the interference of two counterpropagating laser fields, whose linear polarizations are orthogonal (lin $\perp$ lin configuration [21].) We consider a $J_{g}=1 / 2 \rightarrow J_{e}=3 / 2$ atomic transition. This is the simplest atomic transition for which Sisyphus cooling [22] takes place. For this transition, the light interference pattern results into a bipotential $U_{ \pm}$

$$
U_{ \pm}(z)=\frac{U_{0}}{2}[-2 \pm \cos (2 k z)]
$$

for the atoms, with the atom in the ground state sublevel $|+1 / 2\rangle$ experiencing the potential $U_{+}$, while the atoms in the other ground state sublevel, $|-1 / 2\rangle$, will experience $U_{-}$. In Eq. (11), $U_{0}$ is the depth of the optical potential, $z$ the atomic position along the propagation direction of the laser fields, and $k$ the laser field wavevector. Besides the depth $U_{0}$ of the optical potential, another important parameter to characterize the system is the photon scattering rate $\Gamma^{\prime}$. The photon scattering rate from the excited state determines the stochastic optical transitions between the two ground state sublevels, with departure rates given by $\gamma_{ \pm \rightarrow \mp}(z)=\Gamma^{\prime}(1 \pm \cos (2 k z)) / 9$. These transitions lead to a friction force and to fluctuations in the atomic dynamics.

For the study of the ratchet effect, we include a biharmonic driving with frequencies $\omega_{d}, 2 \omega_{d}$, and relative phase between harmonics equal to $\phi$. For an appropriate choice of the phase $\phi$ the drive breaks the relevant time-symmetries of the system, and thus leads to directed motion [23 31].

In addition to the bi-harmonic ac drive, we include a dc bias $F_{d c}$, which corresponds to the load for the motor. The total applied driving force $F(t)$ is thus

$$
F(t)=F_{a c}\left[A_{d} \cos \omega_{d} t+B_{d} \cos \left(2 \omega_{d} t+\phi\right)\right]+F_{d c} .
$$

Here $F_{a c}$ is the amplitude of the ac drive, with relative amplitude between harmonics given by $A_{d}, B_{d}$, and $F_{d c}$ is the amplitude of the applied dc force.

As for the units, throughout this work, the atomic momenta will be expressed in terms of the recoil momentum $p_{r}=\hbar k$ and the energies in terms of the recoil energy $E_{r}=(\hbar k)^{2} / 2 m$, where $m$ is the atomic mass. The scattering rate and the time will be expressed in terms of the recoil angular frequency $\omega_{r}=E_{r} / \hbar$, and its inverse. Finally, the amplitudes of the applied forces will be expressed in terms of $F_{r}=\hbar k \omega_{r}$.

For all the results presented in this work, the following parameters are fixed: $U_{0}=200 \mathrm{E}_{r}$, and $\omega_{d}=\omega_{v}$, where $\omega_{v}$ is the vibrational frequency of the atoms at the bottom of the wells. For the calculations with a bi-harmonic force we take: $A_{d}=B_{d}=1$, while $A_{d}=1, B_{d}=0$ holds when a single harmonic drive is considered for comparison.

\section{NUMERICAL RESULTS}

The numerical simulation techniques are the same as used in previous work [20]. We simulate the atomic dynamics in the optical lattice in the presence of the driving by semi-classical Monte Carlo simulations [21]. A secondorder stochastic Runge-Kutta algorithm with adaptative time-step is used to evolve the atomic position and momentum between jumps corresponding to changes of the internal state. By averaging over the different atomic trajectories, it is then possible to determine the quantities of interest, as the average atomic momentum or the spatial diffusion coefficient, for different choices of parameters. All numerical results presented in this work are producing by taking into account $n=10^{4}$ atomic trajectories. The atoms are initially at the bottom of a well with zero momentum. The drive is then adiabatically turned on during 100 cycles. A further 1000 cycles are then used to calculate the quantities of interest.

The average steady-state atomic momentum is defined as

$$
\langle p\rangle=\left\langle\lim _{t \rightarrow+\infty} \frac{1}{t} \int_{0}^{t} p(t) d t\right\rangle
$$

where \langle\rangle indicates the average over the ensemble. In practice, in the simulation we calculate $\langle p\rangle$ by averaging over the ensemble and over the last 1000 drive cycles, i.e. we calculate $\langle p\rangle=\overline{\langle p(t)\rangle}$, where the ${ }^{-}$sign indicates the timeaverage.

\section{A. Stall force and mobility}

We first address the issue of whether, and to which extent, an ac driven ratchet for cold atoms can operate as a motor. We thus start our analysis by studying the atomic current as a function of the time-symmetry breaking parameter $\phi$ in the presence of a bias force $F_{d c}$. Our results are shown in Fig. 1.

For zero bias the average atomic momentum has a sinlike dependence on the phase $\phi$, as already extensively discussed in previous experimental and theoretical work on rocking ratchets for cold atoms [14 16, 20], as well as in the framework of more general theory 28 30. For non-zero bias, the current still shows a sin-like dependence on the phase $\phi$ but with an additional vertical offset. Actually, as from the inset of Fig. 1, the overall shape of the current as a function of $\phi$ is essentially unchanged, and the applied bias only produces an overall shift. Thus, the average momentum is well described by the relationship: 


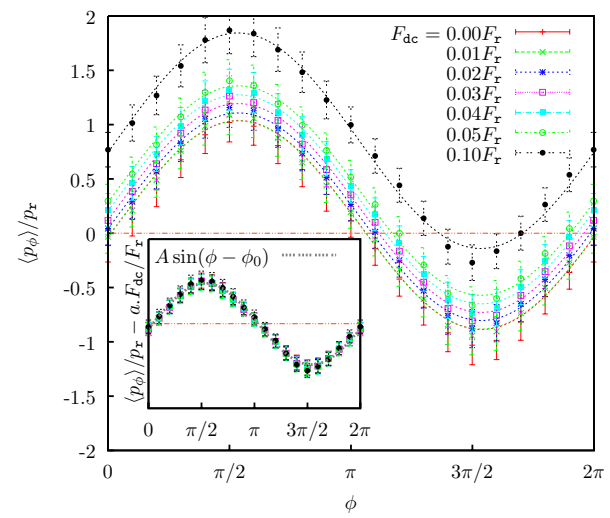

FIG. 1: (Color online) Average atomic momentum as a function of the relative phase $\phi$ between harmonics of the ac drive, for different values of the applied dc bias. The data from our simulations are plotted together with the best fit with the function $\left\langle p_{\phi}\right\rangle / p_{r}=A \sin \left(\phi-\phi_{0}\right)$, where $A$ and $\phi_{0}$ are fitting parameters. The inset shows that by shifting the data by an amount $-\alpha F_{d c} / F_{r}$ proportional to the applied bias force, it is possible to overlap all the curves. Here $\alpha$ is an appropriately chosen constant. This proves that, for the examined range of parameters, the dc bias results in a pure shift of the curve, whose magnitude is proportional to the bias, with no significant distortion in shape. The parameters of the calculation are: $\Gamma^{\prime}=2 \omega_{r}, F_{\mathrm{ac}}=100 F_{r}$.

$$
\frac{\left\langle p_{\phi}\right\rangle}{p_{r}}=A \sin \left(\phi-\phi_{0}\right)+B,
$$

where the phase $\phi_{0}$ corresponds to symmetry-breaking produced by dissipation [16, 29]. Although $\phi_{0}$ is negligeable for the parameters of Fig. 11, it can be large for other sets of parameters, as investigated in detail experimentally and theoretically in previous work [12, 16, 20].

Figure 1 proves that for any phase $\phi \neq \phi_{0}+n \pi$, with $n$ integer, the ratchet can operate as a Brownian motor, i.e. can do work against a load, whose sign and maximum value depends on the phase $\phi$. To characterize further this behaviour, we now define a specific Brownian motor by fixing the Hamiltonian. Specifically, we consider the case of $\phi=3 \pi / 2$, so to break all the relevant symmetries.

A first important parameter which characterizes a Brownian motor is the stall force $F_{\text {stall }}$. This is the largest bias force - or load - against which the motor can operate.

To determine $F_{\text {stall }}$ we study the average atomic momentum $\left\langle p_{3 \pi / 2}\right\rangle$ as a function of the bias force amplitude $F_{d c}$. We obtain in this way the so-called load-velocity curve, as reported in Fig. 2 for different values of the driving strength. The crossing point of the load-velocity curve with the horizontal axis defines the stall force $F_{\text {stall }}$. This is the maximum (in absolute value) load against which the motor can do (positive) work.

The results from Fig. 2 show a linear relationship between the average atomic momentum and the applied dc

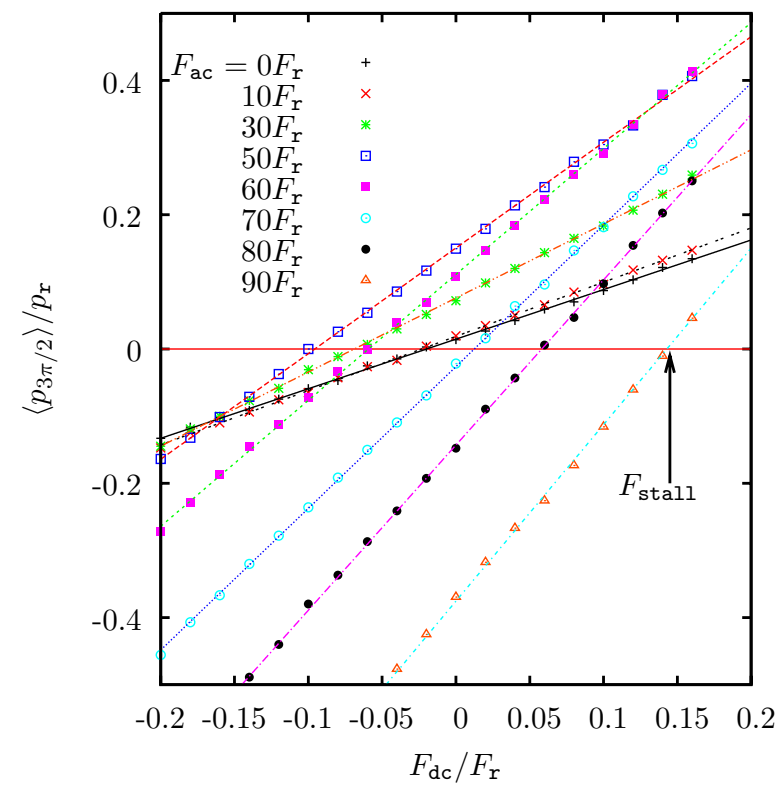

FIG. 2: (Color online) Load-velocity curve for the considered $1 \mathrm{D}$ rocking ratchet for cold atoms. The average atomic momentum is plotted as a function of the applied dc bias, for different amplitudes of the ac drive. The lines are the best linear fits to the numerical data.

bias, i.e. the (differential) mobility $\mu$

$$
\mu=\frac{d\left\langle v\left(F_{d c}\right)\right\rangle}{d F_{d c}}
$$

is independent of $F_{d c}$. Thus, the relationship between the average atomic momentum and the applied dc bias can be well described by:

$$
\frac{\left\langle p_{3 \pi / 2}\right\rangle}{p_{r}}=m \omega_{r} \mu\left(F_{a c}, \Gamma^{\prime}\right)\left(F_{d c}-F_{\text {stall }}\left(F_{a c}, \Gamma^{\prime}\right)\right) / F_{r}
$$

where we evidenced that both $F_{\text {stall }}$ and the mobility $\mu$ depend on the strength of the ac drive as well as on the scattering rate $\Gamma^{\prime}$. The dependence of these parameters on $F_{a c}$ is shown in Fig. 3. The two parameters show a very different dependence on the strength of the driving.

The stall force, which characterizes the range over which the ratchet device can operate as a motor, is a nonmonotonic function of $F_{a c}$ (see Fig. 3), and changes sign for increasing $F_{a c}$. This behaviour is similar to the nonmonotonic dependence of the atomic current on the strength of the ac drive in the absence of a dc bias, as studied in Refs. 14, 20] (see also Fig. 4(a)). In particular, the changes of sign of $F_{\text {stall }}$ correspond to current reversals in the unbiased ratchet. The similarity between the two curves can be simply explained in terms of Eq. 6 , and noticing that for our system the mobility is a positive monotonic function of $F_{a c}$, as discussed now.

The atomic mobility $\mu$ characterizes the atomic velocity in response to a change in dc bias or load as from the 
definition of Eq. [5. Our results of Fig. 3 show that the mobility is a positive increasing function of the strength of the ac drive, i.e. the same change in dc force results in a larger average increment in atomic momentum for a larger ac drive. This behaviour is enhanced at small scattering rate $\Gamma^{\prime}$. As anticipated above, as $\mu$ is a monotonic positive function of $F_{\mathrm{ac}}$, the stall force $F_{\text {stall }}$ and $\left\langle p_{3 \pi / 2}\right\rangle$, related via the mobility - see Eq. 6] shows a similar dependence on $F_{\text {ac }}$.

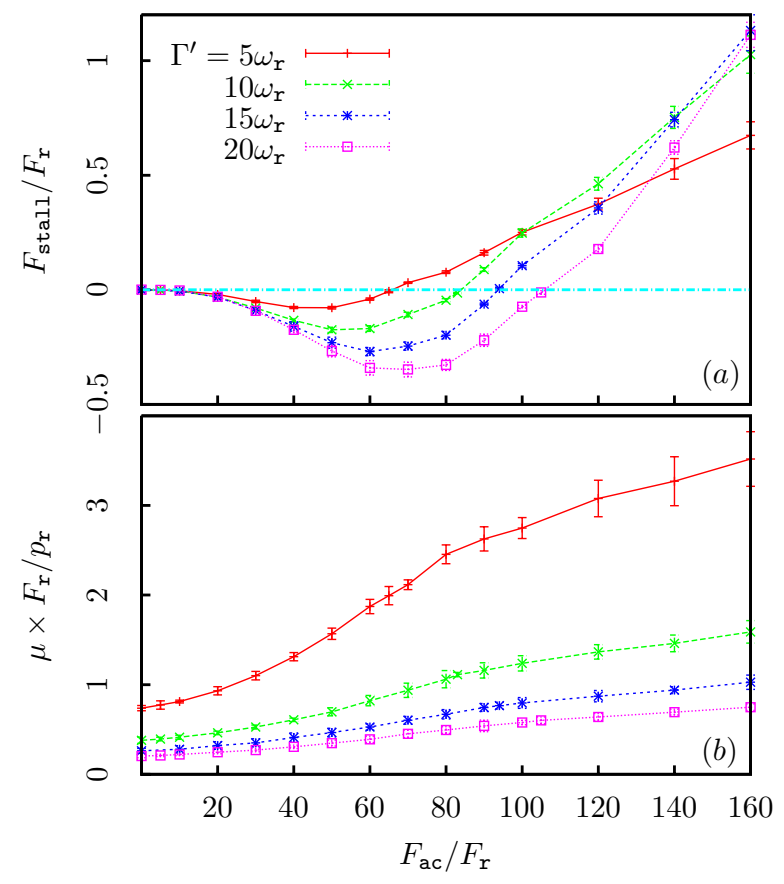

FIG. 3: (Color online) The stall velocity $F_{\text {stall }}(\mathrm{a})$ and the mobility $\mu$ (b), as derived from plots as those in Fig. 2 are plotted as a function of the amplitude of the ac drive, for different values of the scattering rate $\Gamma^{\prime}$.

We also notice that combining Eqs. (4, 6) we obtain an expression for the average atomic momentum as a function of $\phi$ :

$$
\frac{\left\langle p_{\phi}\right\rangle}{p_{r}}=\frac{m \omega_{r} \mu}{\cos \phi_{0}} F_{\text {stall }} \sin \left(\phi-\phi_{0}\right)+m \omega_{r} \mu F_{d c}
$$

which depends only on the mobility $\mu$, the stall force $F_{\text {stall }}$ and the phase-shift $\phi_{0}$ determined by dissipation. It is thus possible to express the atomic current in the general case, i.e. with or without a load, as a function of the parameters $\mu, F_{\text {stall }}$ which fully characterize the operation of the atomic device with a load.

\section{B. Coherency of the atomic transport}

We now turn to the quantitative characterization of transport in an (unbiased) ac driven ratchet. This will then be used to compare ac driven transport to transport induced by an applied dc bias.

The coherency of the directed transport in a ratchet device can be characterized by comparing the linear transport to the spatial spread. Quantitatively, a standard measure [9, 32, 33] of the coherency is the Peclet number

$$
\mathrm{Pe}=\frac{|\langle v\rangle| l}{D_{\mathrm{sp}}} .
$$

Here $l$ is a characteristic length of the system, which in our case will be taken equal to $l=\lambda=2 \pi / k$, twice the spatial period of the optical lattice. $D_{s p}$ is the spatial diffusion coefficient, defined as

$$
D_{\mathrm{sp}}=\lim _{t \rightarrow+\infty} \frac{\left\langle x^{2}(t)\right\rangle-\langle x(t)\rangle^{2}}{2 t} .
$$

Our results for $\langle p\rangle=m\langle v\rangle$, the spatial diffusion coefficient $D_{\mathrm{sp}}$ and the resulting Peclet number are shown in Fig. 4. In (a) the average momentum is plotted as a function of the ac drive amplitude, at different values of the bias force. The regime of small driving, magnified in the inset, was already examined in Refs. [14, 20]. There, the nonmonotonic dependence of the average momentum on the strength of the ac drive was discussed in details, and it was observed that the current first increases (in absolute value) with $F_{a c}$, then decreases and changes sign. Out present study extends well beyond the regime analyzed previously, and shows that beyond the previously observed reversal, the current increase significantly. It then reaches a maximum and start decreasing. The data shows evidence of a second current reversal.

In Fig. 4(b) we report our numerical results for the spatial diffusion coefficients for a driven optical lattice 34]. Our data show that the spatial diffusion coefficient is a rapidly increasing function of the strength of the ac drive, and this behaviour is enhanced at small values of the scattering rate $\Gamma^{\prime}$. By combining the data for the atomic momentum (Fig. 廿4(a)) and for the spatial diffusion coefficient (Fig. 4(b)) we derived the Peclet number which characterizes the coherency of the transport, as plotted in Fig. 4(c). The Peclet number shows two (local) maxima as a function of $F_{\mathrm{ac}}$. The first maximum is observed in the regime of small driving forces. In this regime also the average momentum shows a maximum, i.e. the maximum in the coherency is determined both by the nonmonotonic dependence of the average momentum and by the monotonic increase of the diffusion coefficient. The second maximum of the coherency is observed in the range of forces beyond the first current reversal. In this case around the maximum of the coherence the average momentum is an increasing function of $F_{\mathrm{ac}}$, and the maximum in coherency is due to the faster monotonic increase in the diffusion coefficient. The largest value for the coherency is associated with the second maximum. We notice, however, that although the average momentum for the second maximum is about one order of magnitude larger than the momentum for the first maximum, 


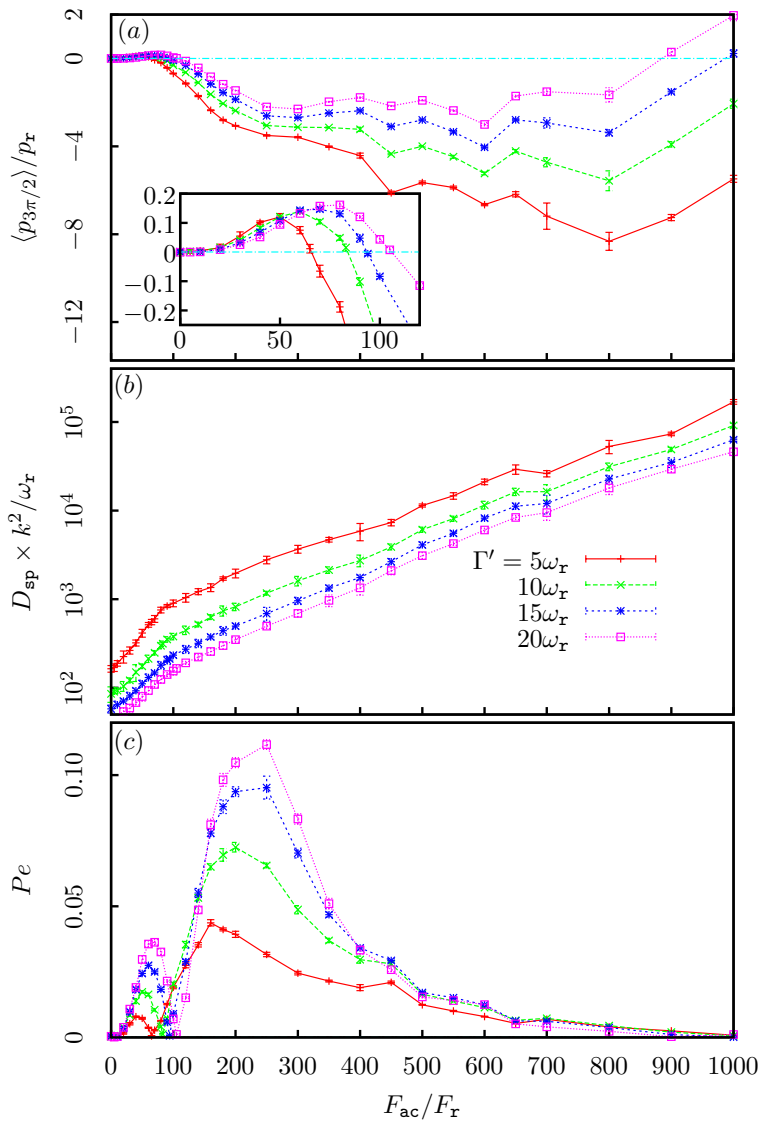

FIG. 4: (Color online) Study of the coherency of the cold atom ratchet. The average atomic momentum (a), the spatial diffusion coefficient (b) and the resulting Peclet number are reported as a function of the amplitude of the ac drive for different values of the scattering rate $\Gamma^{\prime}$. All the data refers to an unbiased system, i.e. $F_{d c}=0$.

the increase in coherency is only of the order of a factor three, because of the large increase in the diffusion coefficient.

\section{Dynamics stabilization}

As final point of our analysis, we aim to compare the characteristics of the transport in the considered 1D rocking ratchet with the features for the transport obtained by simply applying a dc force. We therefore made additional numerical simulations for atoms in an optical lattice in the presence of an applied dc force of amplitude $F_{d c}$, without ac driving $\left(F_{a c}=0\right)$. The results of our numerical calculations are shown in Fig. 5, for two different scattering rates $\Gamma^{\prime}$. In order to be able to compare the coherency for the two set-ups, we plotted the coherency as a function of the generated momentum. In this way, for a given generated momentum we can meaningfully compare the coherences for transport induced by a dc bias and by ac forces.
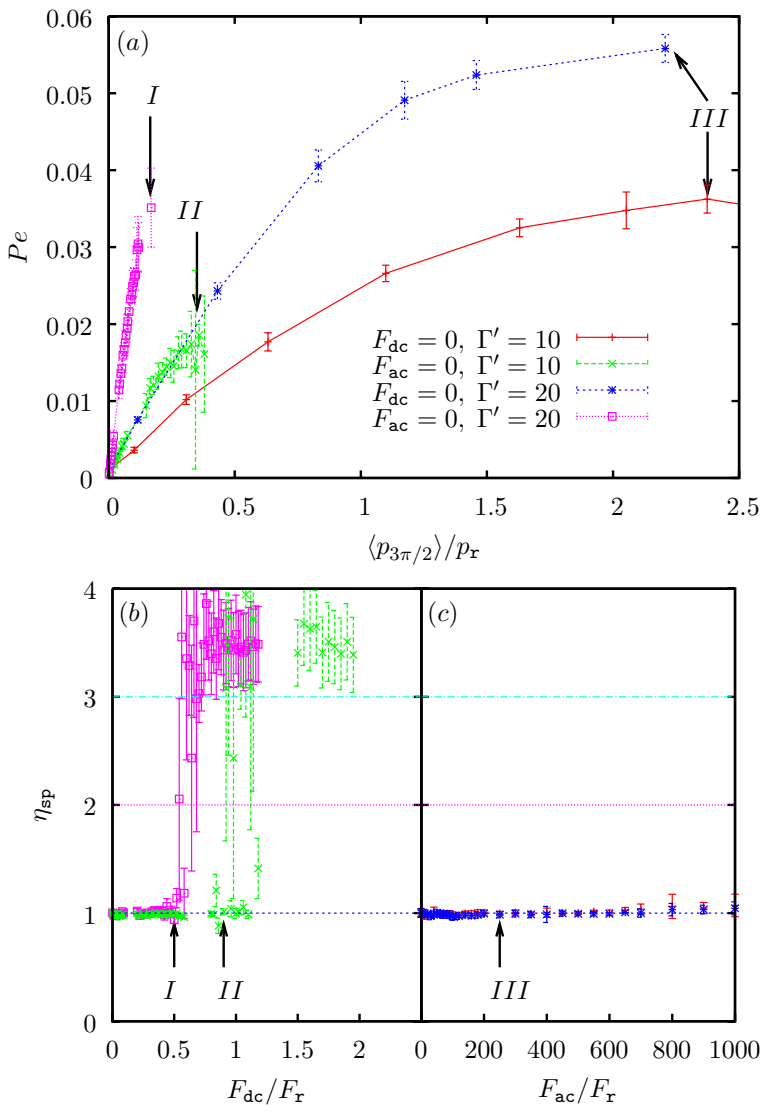

FIG. 5: (Color online) Comparison between the coherency of transport in a cold atom ratchet and the transport produced by applying a dc force. In (a) the Peclet numbers for the trasport produced by an applied dc force to cold atoms in an optical lattice are reported together with the Peclet number for the trasport produced by a applied ac bi-harmonic drive. In both cases the Peclet number is plotted as a function of the obtained average atomic momentum. The data for the acdrive case were generated by considering $F_{a c}$ values from the first current reversal to the maximum of Pe (see Fig. (4). In (b) the spatial diffusion esponent is plotted as a function of the dc force, with $F_{a c}=0$. In (c) the spatial diffusion esponent is plotted as a function of the ac force amplitude, with $F_{d c}=0$. To allow for comparison between (b) and (c) with (a), for each of the curves in (b) and (c) we indicate with an arrow the driving strength (dc and ac, respectively) corresponding to certain values of the atomic momentum which are also reported in (a).

Fig. [5(a) shows that for both the considered values of $\Gamma^{\prime}$, the transport induced by the dc bias shows a larger coherency than the one generated by the ratchet effect. This is due to the fact that, as we verified numerically, for a dissipative optical lattice of the type considered here an applied dc bias does not modify significantly the diffusion coefficient [35], at variance with the large increase produced by an ac driving. Thus, a given average atomic momentum can be induced with a larger coherency by a dc force rather than by an ac driving. However, as an 
important point, we notice that by applying a dc force it is possible to generate uniform motion only over a limited range of atomic momentum, and this is the reason why the curves in Fig. 5 corresponding to the dc-bias case extend only up to a certain value of the atomic momentum. Beyond that value, the motion becomes accelerated. To verify this point, we determined from our data the diffusion exponent $\eta_{s p}$ defined as

$$
\left\langle x^{2}(t)\right\rangle-\langle x(t)\rangle^{2} \sim t^{\eta_{s p}}
$$

in the limit $t \rightarrow \infty$. According to the definition, $\eta_{s p}=1$ corresponds to normal diffusion, and $\eta_{s p}=4$ to uniformly accelerated motion. It is therefore a sensitive quantity to detect persistent accelerated motion. Our results for the diffusion exponent are reported in Fig. 55(b) and (c) for the case of dc and ac driving, respectively. Fig. [5) shows clearly that the application of a dc bias beyond a certain crytical value results into a crossover to accelerated motion, characterized by $\eta_{s p}=4$. The actual numerical value is somewhat smaller than $\eta_{s p}=4$ for a finite time window indicating that not all the atoms may be accelerated just above the threshold. In order to understand this behaviour, it is sufficient to recall that the damping mechanism associated to Sisyphus cooling has a finite capture range [22]. Thus, above a certain threshold an applied dc force will accelerate most of the atoms. This behaviour has to be contrasted to the dynamics produced by ac forces. Figure 5(a) shows that the use of an ac drive allows the generation of uniform motion over a large range of momentum, much larger than what possible with a dc bias. This is confirmed by Fig. 5(c) which shows that the diffusion is normal over a very broad range of applied ac forces. Thus, we observe a surprising phenomenon: the bi-harmonic ac force stabilize the dynamics, allowing the generation of uniform directed motion over a range of momentum much larger than what possible with a dc bias.

To explain such a stabilization of the dynamics, we refer to Fig. 6] where the ensemble-averaged timedependent atomic momentum is studied for three different situations: dc drive, bi-harmonic driving, and single harmonic drive. The dc drive case also includes a calculation for an unbiased system for comparison. The time-derivative of the total atomic energy

$$
E=\frac{p^{2}}{2 m}+U_{ \pm}(x)
$$

is also reported for the three cases as a function of the atomic momentum, to identify the ranges of atomic momentum in which dissipation takes place. The derivative $\langle d E(p) / d t\rangle$ is calculated from the atomic trajectories in the following way. For a given mometum $p$ we identify in the considered $n$ atomic trajectories the $N(p)$ instants $t_{i}(i=1 \ldots N)$ at which an atom has a momentum $p$. We then determine the energy increment $\Delta E_{i}$ following the (variable step-size) increment $d t_{i}$. The time-derivative is
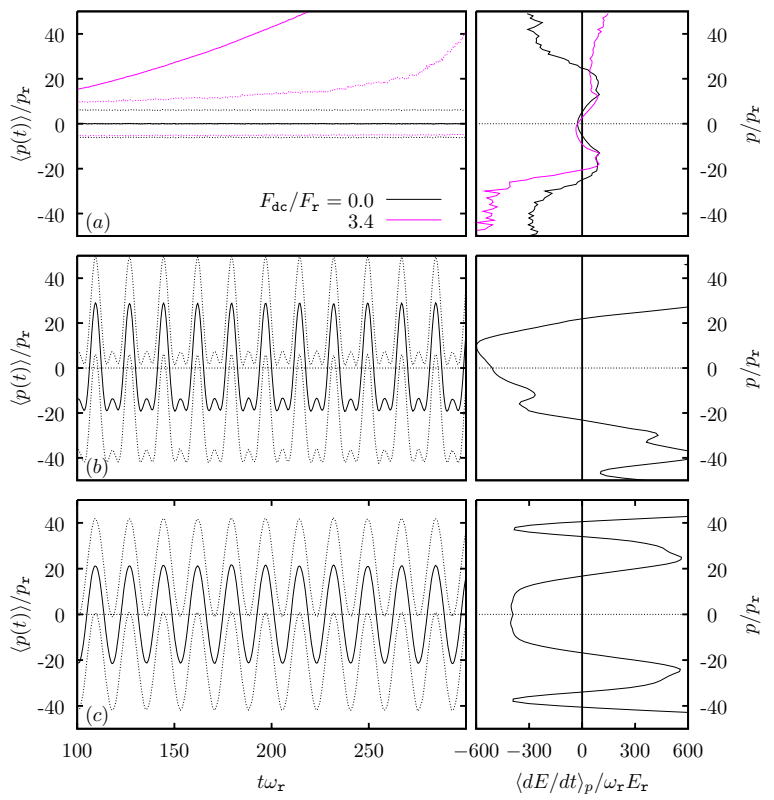

FIG. 6: (Color online) Ensemble-averaged time-dependent atomic momentum (solid line) as a function of time. Left: In (a) the case of a dc drive with $F_{d c}=3.4 F_{r}$ is considered together with the unbiased case $F_{d c}=0$; (b) refers to a bi-harmonic drive with $F_{a c}=600 F_{r}$. Finally, (c) report the data for a single-harmonic drive with $F_{a c}=600 F_{r}$. In all cases the dashed lines indicate the instanteneous interval $\left[p_{-}: p_{+}\right]$which contains $68 \%$ of the momentum distribution, with $P\left(p>p_{+}\right)=P\left(p<p_{-}\right)=16 \%$. We notice that for (a) $p_{-}$is, within the numerical accuracy, the same for the two considered cases. Right: The plots show the timeaverage derivative of the total atomic energy as a function of the atomic momentum.

then given by

$$
\langle d E(p) / d t\rangle=\frac{1}{N(p)} \sum_{i=1}^{N(p)} \frac{\Delta E_{i}}{d t_{i}} .
$$

This quantity is the average power input of the external forces, noise and internal state transitions which are responsible for the cooling and heating processes. Consider first the case of an unbiased and undriven system, as in Fig. 6(a). We can distinguish a range of momentum in which energy is in average dissipated (cooling region): $\langle d E(p) / d t\rangle<0$, and a heating region in which energy increases. At steady state, the atomic momentum distribution extends over both regions, so that the average energy is constant. We notice that atoms explore the heating region to then return to the cooling region, i.e. there is a continuous interchange of atoms between the two regions. Consider now the application of a dc force. The corresponding time-derivative of the energy is then modified by a term which includes the power supplied by the bias. As from Fig. 6(a) beyond a certain threshold (as identified in Fig. [5), the atoms are accelerated. This is because essentially a large fraction of the momentum 
distribution is now located in the heating region, and the atoms can persist there. As a given atom trajectory does not cover the cooling region, energy is not dissipated and the steady-state energy balance is lost. Accelerated motion is thus produced.

Consider now the application of ac drivings. Both cases of bi-harmonic and single-harmonic drive are studied in Fig. [6. (b) and (c), respectively. As already obseved, bi-harmonic drive allows the generation of uniform motion over a range of momentum much larger than in the case of a dc bias. Such a surprising stabilization of the dynamics can be explained as a result of two contributions. First, an ac drive broadens the effective cooling region. Second, for an ac drive the time-dependent atomic momentum oscillates in time. This forces the atom trajectories to cover the cooling region. Thus the system can dissipate energy and maintain an energy balance. As from Fig. 6 (b) and (c), this holds for both bi-harmonic and single-harmonic drivings. Furthermore, for a bi-harmonic drive the energy balance allows for the generation of directed motion at constant average velocity over a broad range of atomic momentum.

\section{CONCLUSIONS}

In conclusion, in this work we analyzed two aspects of ac driven transport in cold atoms ratchets. We first addressed the issue of whether, and to which extent, an ac driven ratchet for cold atoms can operate as a motor. We thus studied and characterize theoretically the motor corresponding to a $1 \mathrm{D}$ rocking ratchet for cold atoms, as obtained by adding a load to the ratchet set-up. We demonstrated that a current can be generated also in the presence of a load, e.g. the ratchet device can operate as a motor. Correspondingly, we determined the stall force for the motor, which characterizes the range of loads over which the device can operate as a motor, and the mobility, which describes the sensitivity to changes in the load magnitude. We then compared our results for the transport in an ac driven ratchet device with the transport in a dc driven system. We observed a peculiar phenomenon: the bi-harmonic ac force stabilizes the dynamics, allowing the generation of uniform directed motion over a range of momentum much larger than what possible with a dc bias. We explained such a stabilization of the dynamics by observing that a non-adiabatic ac drive broadens the effective cooling momentum range, and forces the atom trajectories to cover such a region. Thus the system can dissipate energy and maintain an energy balance. This allows for the generation of directed motion at constant average velocity over a broad range of atomic momentum.

As an interesting point, our results show that in the case of a finite-range velocity-dependent friction, a ratchet device may offer the possibility of controlling the motion over a broader range of momentum with respect to a purely biased system, although this is at the cost of a reduced coherency.

\section{ACKNOWLEDGEMENTS}

We thank the Royal Society and the Leverhulme Trust for financial support.
[1] A. Ajdari and J. Prost, C.R. Acad. Sci. Paris 315, 1635 (1992).

[2] M.O. Magnasco, Phys. Rev. Lett. 71, 1477 (1993).

[3] A. Adjari, D. Mukamel, L. Peliti, J. Prost, J. Phys. I (France) 4, 1551 (1994).

[4] R. Bartussek, P. Hänggi and J.G. Kissner, Europhys. Lett. 28, 459 (1994).

[5] C.R. Doering, W. Horsthemke, and J. Riordan, Phys. Rev. Lett. 72, 2984 (1994).

[6] P. Reimann, Phys. Rep. 361, 57 (2002);

[7] P. Hänggi and F. Marchesoni, Rev. Mod. Phys. 81, 387 (2009).

[8] R. Krishnan, M.C. Mahato, and A.M. Jayannavar, Phys. Rev. E 70, 021102 (2004).

[9] H. Linke, M.T. Downton, and M.J. Zuckermann, Chaos 15, 026111 (2005).

[10] M. Kostur, L. Machura, P. Hänggi, J. Luczka, and P. Talkner, Physica A 371, 20 (2006).

[11] A.V. Ponomarev, S. Denisov, and P. Hänggi, Phys. Rev. Lett. 102, 230601 (2009).

[12] F. Renzoni, Adv. At. Mol. Opt. Phys. 57, 1 (2009).

[13] P. Sjolund, S.J.H. Petra, C.M. Dion, S. Jonsell, M. Nylen, L. Sanchez-Palencia, A. Kastberg, Phys. Rev. Lett. 96, 190602 (2006); P. Sjolund, S.J.H. Petra, C.M. Dion,
H. Hagman H, S. Jonsell, A. Kastberg, Eur. Phys. J. D 44, 381 (2007); H. Hagman, C.M. Dion, P. Sjolund, S.J.H. Petra, A. Kastberg A, EPL 81, (33001) 2008.

[14] P.H. Jones, M. Goonasekera, and F. Renzoni, Phys. Rev. Lett. 93, 073904 (2004).

[15] R. Gommers, P. Douglas, S. Bergamini, M. Goonasekera, P.H. Jones, and F. Renzoni, Phys. Rev. Lett. 94, 143001 (2005).

[16] R. Gommers, S. Bergamini, and F. Renzoni, Phys. Rev. Lett. 95, 073003 (2005).

[17] R. Gommers, S. Denisov and F. Renzoni, Phys. Rev. Lett. 96, 240604 (2006); R. Gommers, M. Brown, and F. Renzoni, Phys. Rev. A 75, 053406 (2007).

[18] R. Gommers, V. Lebedev, M. Brown, and F. Renzoni, Phys. Rev. Lett. 100, 040603 (2008).

[19] V. Lebedev and F. Renzoni, Phys. Rev. A 80, 023422 (2009).

[20] M. Brown and F. Renzoni, Phys. Rev. A 77, 033405 (2008).

[21] G. Grynberg and C. Mennerat-Robilliard, Phys. Rep. 355, 335 (2001).

[22] J. Dalibard and C. Cohen-Tannoudji, J. Opt. Soc. Am. B6, 2023 (1989).

[23] F. Marchesoni, Phys. Lett. A 119, 221 (1986). 
[24] M.C. Mahato and A.M. Jayannavar, Phys. Lett. A 209, 21 (1995).

[25] D.R. Chialvo and M.M. Millonas, Phys. Lett. A 209, 26 (1996).

[26] M.I. Dykman, H. Rabitz, V.N. Smelyanskiy, and B.E. Vugmeister, Phys. Rev. Lett. 79, 1178 (1997).

[27] I. Goychuk and P. Hänggi, Europhys. Lett. 43, 503 (1998).

[28] S. Flach, O. Yevtushenko and Y. Zolotaryuk, Phys. Rev. Lett. 84, 2358 (2000).

[29] O. Yevtushenko, S. Flach, Y. Zolotaryuk, A.A. Ovchinnikov, Europhys. Lett. 54, 141 (2001).

[30] P. Reimann, Phys. Rev. Lett. 86, 4992 (2001).

[31] S. Denisov, Y. Zolotaryuk, S. Flach, and O. Yevtushenko, Phys. Rev. Lett. 100, 224102 (2008).
[32] L. Machura, M. Kostur, F. Marchesoni, P. Talkner, P. Hänggi and J. Luczka, J. Phys.: Condens. Matter 17, S3741 (2005).

[33] S. Roy, D. Dan and A.M. Jayannavar, J. Stat. Mech. P09012, (2006).

[34] For a study of spatial diffusion in undriven optical lattices, see: F.-R. Carminati, M. Schiavoni, L. SanchezPalencia, F. Renzoni and G. Grynberg, Eur. Phys. J. D 17, 249 (2001).

[35] This behaviour is specific to the examined dissipative optical lattice. In other cases a dc bias may modify significantly the diffusion coefficient, as derived in P. Reimann, C. Van den Broeck, H. Linke, P. Hänggi, J.M. Rubi, and A. Pérez-Madrid, Phys. Rev. Lett. 87, 010602 (2001). 